ARTICLE

\author{
Received 30 Dec 2014 | Accepted 16 Mar 2015 | Published 24 Apr $2015 \quad$ DOl: 10.1038/ncomms7926 OPEN
}

\title{
Mechanisms of splicing-dependent trans-synaptic adhesion by PTP $\delta$-IL1RAPL1/IL-1RAcP for synaptic differentiation
}

\author{
Atsushi Yamagata1,2,3, Tomoyuki Yoshida4,5,6, Yusuke Sato1,2,3, Sakurako Goto-Ito1, Takeshi Uemura3,5,7,8, \\ Asami Maeda1,3, Tomoko Shiroshima1,3, Shiho Iwasawa-Okamoto4,6, Hisashi Mori ${ }^{4}$, Masayoshi Mishina5,9 \\ \& Shuya Fukai $1,2,3$
}

Synapse formation is triggered through trans-synaptic interaction between pairs of pre- and postsynaptic adhesion molecules, the specificity of which depends on splice inserts known as 'splice-insert signaling codes'. Receptor protein tyrosine phosphatase $\delta$ (PTP $\delta$ ) can bidirectionally induce pre- and postsynaptic differentiation of neurons by trans-synaptically binding to interleukin-1 receptor accessory protein (IL-1RAcP) and IL-1RAcP-like-1 (IL1RAPL1) in a splicing-dependent manner. Here, we report crystal structures of PTP $\delta$ in complex with IL1RAPL1 and IL-1RAcP. The first immunoglobulin-like (Ig) domain of IL1RAPL1 directly recognizes the first splice insert, which is critical for binding to IL1RAPL1. The second splice insert functions as an adjustable linker that positions the lg2 and Ig3 domains of PTP $\delta$ for simultaneously interacting with the Ig1 domain of IL1RAPL1 or IL-1RACP. We further identified the ILIRAPL1-specific interaction, which appears coupled to the first-splice-insert-mediated interaction. Our results thus reveal the decoding mechanism of splice-insert signaling codes for synaptic differentiation induced by trans-synaptic adhesion between PTP $\delta$ and ILIRAPL1/IL-1RACP.

\footnotetext{
${ }^{1}$ Structural Biology Laboratory, Life Science Division, Synchrotron Radiation Research Organization and Institute of Molecular and Cellular Biosciences, The University of Tokyo, Tokyo 113-0032, Japan. ${ }^{2}$ Department of Medical Genome Sciences, Graduate School of Frontier Sciences, The University of Tokyo, Chiba 277-8501, Japan. ${ }^{3}$ CREST, JST, Saitama 332-0012, Japan. ${ }^{4}$ Department of Molecular Neuroscience, Graduate School of Medicine and Pharmaceutical Sciences, University of Toyama, Toyama 930-0194, Japan. ${ }^{5}$ Department of Molecular Neurobiology and Pharmacology, Graduate School of Medicine, The University of Tokyo, Tokyo 113-0033, Japan. ${ }^{6}$ PRESTO, JST, Saitama 332-0012, Japan. 7 Department of Molecular and Cellular Physiology, Shinshu University School of Medicine, Nagano 390-8621, Japan. ${ }^{8}$ Institute for Biomedical Sciences, Interdisciplinary Cluster for Cutting Edge Research, Shinshu University, Nagano 390-8621, Japan. ${ }^{9}$ Brain Science Laboratory, The Research Organization of Science and Technology, Ritsumeikan University, Shiga 525-8577, Japan. Correspondence and requests for materials should be addressed to T.Y. (email: toyoshid@med.u-toyama.ac.jp) or to S.F. (email: fukai@iam.u-tokyo.ac.jp).
} 
M ammalian brains are composed of at least a few hundred billions of neurons, which are connected by synapses in a spatiotemporally organized manner to establish higher-order brain functions such as learning, memory and emotion. Synapse formation is triggered through transsynaptic interactions between selective pairs of pre- and postsynaptic adhesion molecules known as 'synaptic organizers' or 'synaptogenic proteins', many of which are associated with neurodevelopmental disorders such as intellectual disability and autism $^{1-6}$. Recent studies have highlighted a major role of typeIIa receptor protein tyrosine phosphatases (RPTPs) as synaptic organizers in pre- and postsynaptic differentiation during neural development ${ }^{5,6}$. Vertebrate type-IIa RPTPs comprise LAR, PTP $\sigma$ and $\operatorname{PTP} \delta$, which share common domain architecture with a single transmembrane (TM) helix flanked by a large extracellular domain (ECD) and cytoplasmic tandem PTP domains: the membrane-proximal PTP domain (D1) is catalytically active, whereas the membrane-distal PTP domain (D2) is inactive. The ECD consists of three immunogloblin-like (Ig) domains and four to eight fibronectin type-III (Fn) domains. In mammals, presynaptic type-IIa RPTPs form trans-synaptic adhesion by specifically binding to their cognate postsynaptic organizers through their ECDs for inducing synaptic differentiation of neurons. To date, five different types of postsynaptic organizers for type-IIa RPTPs have been reported: interleukin-1 receptor (IL-1R) accessory protein (IL-1RAcP) ${ }^{7}$, IL-1RAcP-like-1 $(\text { IL1RAPL1 })^{8}$, neurotrophin receptor tyrosine kinase C $(\operatorname{TrkC})^{9}$, netrin-G ligand-3 (NGL-3) $)^{10,11}$ and Slit- and Trk-like (Slitrk) family proteins ${ }^{12,13}$. For example, co-culture with either IL-1RAcP- or IL1RAPL1-expressing non-neuronal HEK293T cells can stimulate presynaptic differentiation in contacting axons of cultured cortical neurons, however, the IL1RAPL1and IL-1RAcP-induced presynaptic differentiations are completely abolished and decreased by $\sim 70 \%$ in the neurons lacking PTP $\delta$, respectively ${ }^{7,8}$. Similarly, $\mathrm{PTP} \delta$ can induce postsynaptic differentiation of cultured cortical neurons in a manner specific to IL1RAPL1 and IL-1RAcP (more precisely, a central nervous system-restricted IL-1RAcP isoform, which differs only in the carboxy (C)-terminal from a widely distributed isoform). On the other hand, Slitrk family proteins induce presynaptic differentiation through the interaction with type-IIa RPTPs ${ }^{12,13}$. Among Slitrk family proteins, Slitrk3 selectively induces inhibitory synapse formation, suggesting potential roles of synapse organizers in balancing excitatory and inhibitory synapses ${ }^{12}$. Various combinations of trans-synaptic adhesion between pre- and postsynaptic organizers might play an important role in generating extremely diverged but highly organized synaptic connections of neurons.

Varieties of the combinations between pre- and postsynaptic organizers rely upon structural diversities of their ECDs, which can be drastically increased by multiple isoforms and splice variants. In fact, several isoforms and splice variants of type-IIa RPTPs are expressed in mouse brain, and bind selectively to the corresponding postsynaptic organizers ${ }^{5,6}$. Type-IIa RPTPs commonly have three Ig domains $(\operatorname{Ig} 1-\operatorname{Ig} 3)$ in their amino (N)-terminal regions. The first splicing site is located within Ig2, whereas the second one is in the junction between Ig2 and Ig3. These two splicing sites generate variants of the type-IIa RPTPs that lack or contain short-peptide inserts termed mini-exon peptides. A recent study has shown that Slitrk family members bind to all type-IIa RPTP members, depending on the second mini-exon peptide $(\mathrm{meB})^{14}$. On the other hand, IL1RAPL1 and IL-1RAcP specifically bind to PTP $\delta$ but not to LAR or PTP $\sigma$. Furthermore, our previous cell-surface binding assays showed that both the first mini-exon peptide (meA) and meB can alter their binding properties ${ }^{7,8}$. MeB comprises four residues,
Glu-Leu-Arg-Glu, whereas there are three variations in meA: a three-residue peptide Glu-Ser-Ile (meA3), a six-residue peptide Gly-Gly-Thr-Pro-Ile-Arg (meA6) and their tandem combination (Glu-Ser-Ile-Gly-Gly-Thr-Pro-Ile-Arg) (meA9). Binding to IL-1RAcP is significantly enhanced by the presence of both $\mathrm{meA}$ and $\mathrm{meB}$, independently of the meA variation. In contrast, the length and sequence of meA are critically important for binding to IL1RAPL1. Only PTP $\delta$ variants containing meA9 and meA6 can bind to IL1RAPL1. The meB insertion increases binding of the meA9-containing variant, whereas the meA6containing variant absolutely requires the combination with meB. These findings indicate that information of synaptogenic postsynaptic ligands for the type-IIa RPTPs is encoded in their splice inserts. Similarly, in another presynaptic organizer neurexin, the insert in the splice site 4 regulates the specificity to postsynaptic organizers Cbln1-GluR $\delta 2$ and LRRTM1/2 (refs 15,16). The 'splice-insert signaling code' concept for specific synaptic connections was proposed originally for transsynaptic adhesion between neurexin and neuroligin, but appears likely more general for synaptogenic adhesion ${ }^{3,7-9,15-18}$. However, structural mechanisms underlying this concept remain elusive. To elucidate the structural basis for decoding the 'splice-insert signaling code' in the type-IIa RPTPs, we determined the crystal structures of PTP $\delta$-ECD in complex with IL-1RAcP-ECD and IL1RAPL1-ECD. Together with structurebased mutational analyses by surface plasmon resonance (SPR) spectroscopy and synaptogenic assays, we reveal the structural basis of the splicing-dependent trans-synaptic adhesion by PTP $\delta$ and IL1RAPL1/IL-1RAcP for synaptic differentiation.

\section{Results}

Structure of the complex between PTP $\delta$ and IL1RAPL1. To elucidate the structural basis of the PTP $\delta$-IL1RAPL1 interaction for trans-synaptic adhesion, we determined the crystal structures of IL1RAPL1-ECD in complex with PTP $\delta$ Ig1-Ig2 and the fulllength PTP $\delta$-ECD at 2.7 and $4.4 \AA$ resolutions, respectively (Fig. 1a,b, Table 1). Description of PTP $\delta$ Ig1-Ig2 will be based on the 2.7 - $\AA$-complex structure, whereas that of the other regions of PTP $\delta$-ECD will be based on the 4.4- $\AA$-complex structure. For crystallography, we used a $\mathrm{PTP} \delta$ isoform containing four Fn domains and both meA9 and meB, which is the most abundant in the developing brain and has the highest affinity for IL1RAPL1 $\left(K_{\mathrm{d}}: 0.15 \mu \mathrm{M}\right)$ among all the PTP $\delta$ variants (Table 2). Hereafter, 'PTP $\delta$ ' indicates this isoform, unless otherwise noted. The Ig1-Ig2 domains of PTP $\delta$ retain the binding ability for IL1RAPL1-ECD, as shown in our serial deletion analysis of PTP $\delta$ (Supplementary Fig. 1a). PTP $\delta$-ECD binds to IL1RAPL1-ECD at a ratio of $1: 1$ and the asymmetric unit contains one PTP $\delta$ IL1RAPL1 complex (Fig. 1b and Supplementary Fig. 1b). IL1RAPL1-ECD consists of three Ig domains, which are arranged in an L-shape (Fig. 1a,b). PTP $\delta$-ECD exhibits an elongated shape with a length of $180 \AA$ along the longest axis. The Ig1 and Ig2 domains of PTP $\delta$ tightly interact with each other to form a compact V-shaped unit, which is almost identical to the isolated Ig1-Ig2 structures of $\mathrm{PTP} \delta^{19}$ (PDB:2YD6, 2YD7) (rmsd values of 0.8-0.9 $\AA$ for $195 \mathrm{C} \alpha$ atoms). No substantial change in the backbone structure of PTP $\delta$ Ig1-Ig2 occurred upon binding to IL1RAPL1 (Supplementary Fig. 1c). The Ig3 domain is spatially separated by the meB-containing linker and positioned apart from the Ig1-Ig2 unit. The following Fn1 and Fn2 domains are aligned in a straight line, similarly to the isolated PTP $\delta$ Fn1-Fn2 structure, which we determined at $2.0 \AA$ resolution (Supplementary Fig. 1d). The Fn3 domain of PTP $\delta$ is oriented perpendicular to the Fn1-Fn2 domains, as observed in the PTP $\sigma$ Ig1-Fn3 structure (Supplementary Fig. 1e). Electron density of the Fn4 domain and subsequent C-terminal 


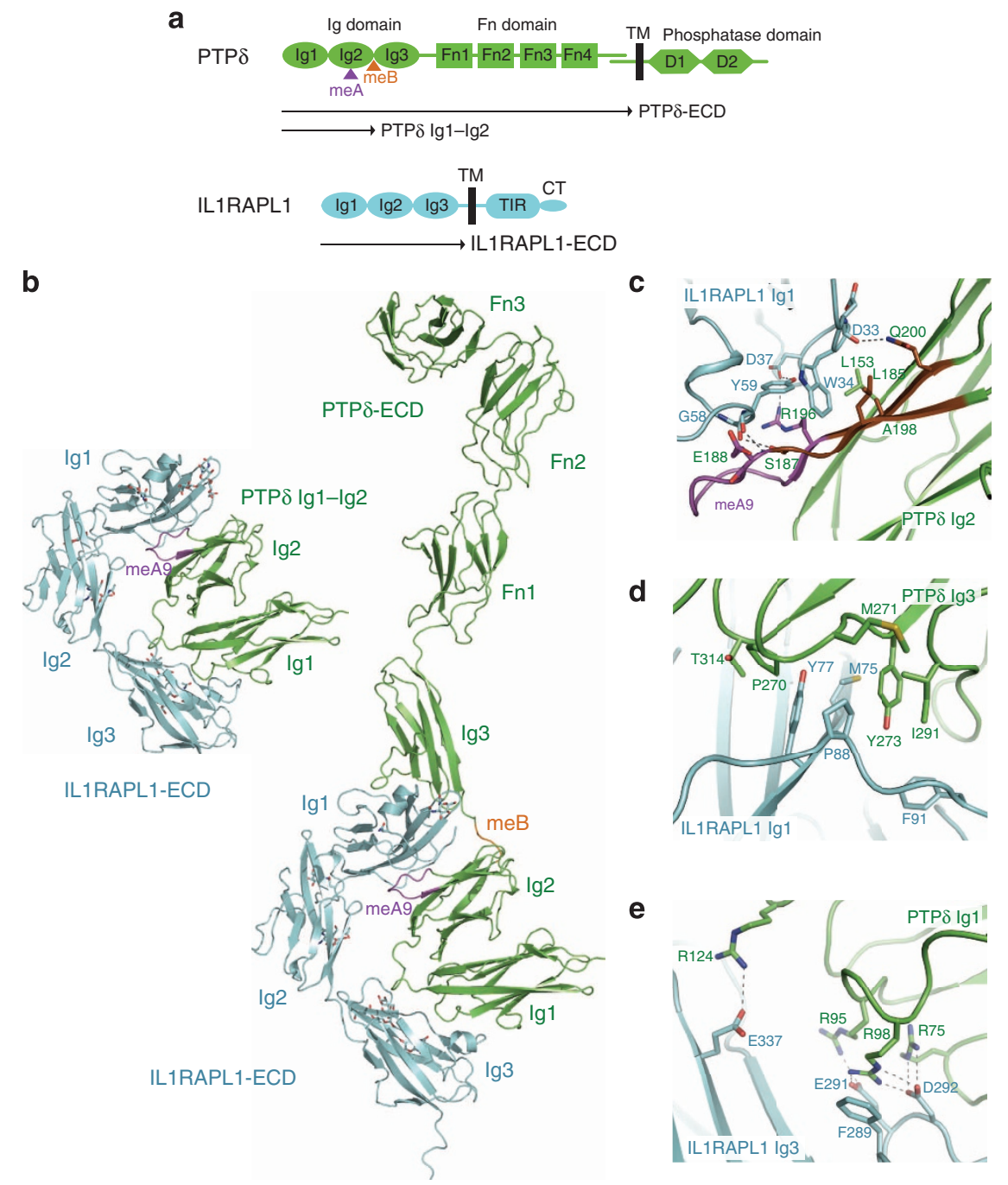

Figure 1 | Structure of the complex between PTP $\delta$ and IL1RAPL1. (a) Domain organizations of PTP $\delta$ and IL1RAPL1. The meA and meB insertion sites of PTP $\delta$ are indicated by magenta and orange triangles, respectively. (b) Overall structures of the PTP $\delta$ Ig1-Ig2•|L1RAPL1-ECD and PTP $\delta$-ECD•|L1RAPL1-ECD complexes. PTP $\delta$ and IL1RAPL1 are coloured green and cyan, respectively. The meA9 and meB insertions are highlighted in magenta and orange, respectively. $\mathrm{N}$-linked glycans are shown as sticks. (c) Interactions between the Ig1 domain of IL1RAPL1 and the Ig2 domain of PTP . The colouring scheme is the same as that in $\mathbf{b}$, except that IL-1RAcP/IL1RAPL1-Ig1-interacting $\beta$-strands of PTP $\delta$ are coloured brown. Residues involved in the interactions are shown as sticks. Hydrogen bonds are indicated by dotted lines. (d) Hydrophobic interactions between the Ig1 domain of IL1RAPL1 and the Ig3 domain of PTP $\delta$. The representation scheme is the same as that in $\mathbf{c}$. (e) Interactions between the Ig3 domain of IL1RAPL1 and the Ig1 domain of PTP $\delta$. The representation scheme is the same as that in $\mathbf{c}$.

region was not visible, likely due to the disorder. The length of the PTP $\delta$-ECD॰IL1RAPL1-ECD complex along the longest axis is $206 \AA$, which is equivalent to the average length of excitatory synaptic clefts, implying that PTP $\delta$-ECD is kinked at the junction between the Fn2 and Fn3 domains in synapses.

Interactions between IL1RAPL1 Ig1 and PTPS Ig2 domains. The Ig1 domain of IL1RAPL1 interacts with both Ig2 and Ig3 domains of PTP $\delta$ (Fig. 1b). The interface between the IL1RAPL Ig1 and PTP $\delta$ Ig2 domains comprises both hydrophobic and hydrophilic interactions with a buried surface area of $699 \AA^{2}$. These interactions occur on two $\beta$-strands comprising Arg181Ser187 and Arg196-Glu202 in the Ig2 domain of PTP $\delta$, between which meA9 is inserted (Fig. 1c). These two $\beta$-strands are hereafter referred to as the IL1RAPL1/IL-1RAcP-Ig1interacting $\beta$-strands, because they also mediate the interaction with the Ig1 domain of IL-1RAcP as described later. The first eight residues of meA9 ( ${ }^{188} \mathrm{Glu}-\mathrm{Ser}-\mathrm{Ile}-\mathrm{Gly}-\mathrm{Gly}$-Thr-Pro-Ile $\left.{ }^{195}\right)$ form a loop connecting these two $\beta$-strands, whereas the last residue, Arg196, extends one of the two $\beta$-strands. Trp34 of IL1RAPL1 hydrophobically interacts with Leu153, Ala198 and Leu185 of PTP $\delta$ (Fig. 1c). The affinity of the IL1RAPL1 W34A mutant for PTP $\delta$ could not be measured by surface-plasmon resonance (SPR) spectroscopy, due to substantially low signals, indicating that this hydrophobic interaction is essential for binding between PTP $\delta$ and IL1RAPL1. The aliphatic portion of PTP $\delta$ Arg196 also participates in this hydrophobic interaction, which enables the Ig2 domain of PTP $\delta$ to more tightly interact with the Ig1 domain of IL1RAPL1 (Fig. 1c). The Arg196 side chain forms a hydrogen bond with Asp37 of IL1RAPL1, which is further stabilized by a hydrogen bond with Tyr59 of IL1RAPL1. Concomitantly, the side chain of Ser187 and the main-chain N atom of Glu188 in meA9 hydrogen bond with the main-chain $\mathrm{O}$ atoms of IL1RAPL1 Tyr59 and Gly58, respectively. The interactions mediated by Arg196 of PTP $\delta$ are critically important: the R196A mutation of PTP $\delta$ and the D37A mutation of 
Table 1 | Data collection and refinement statistics.

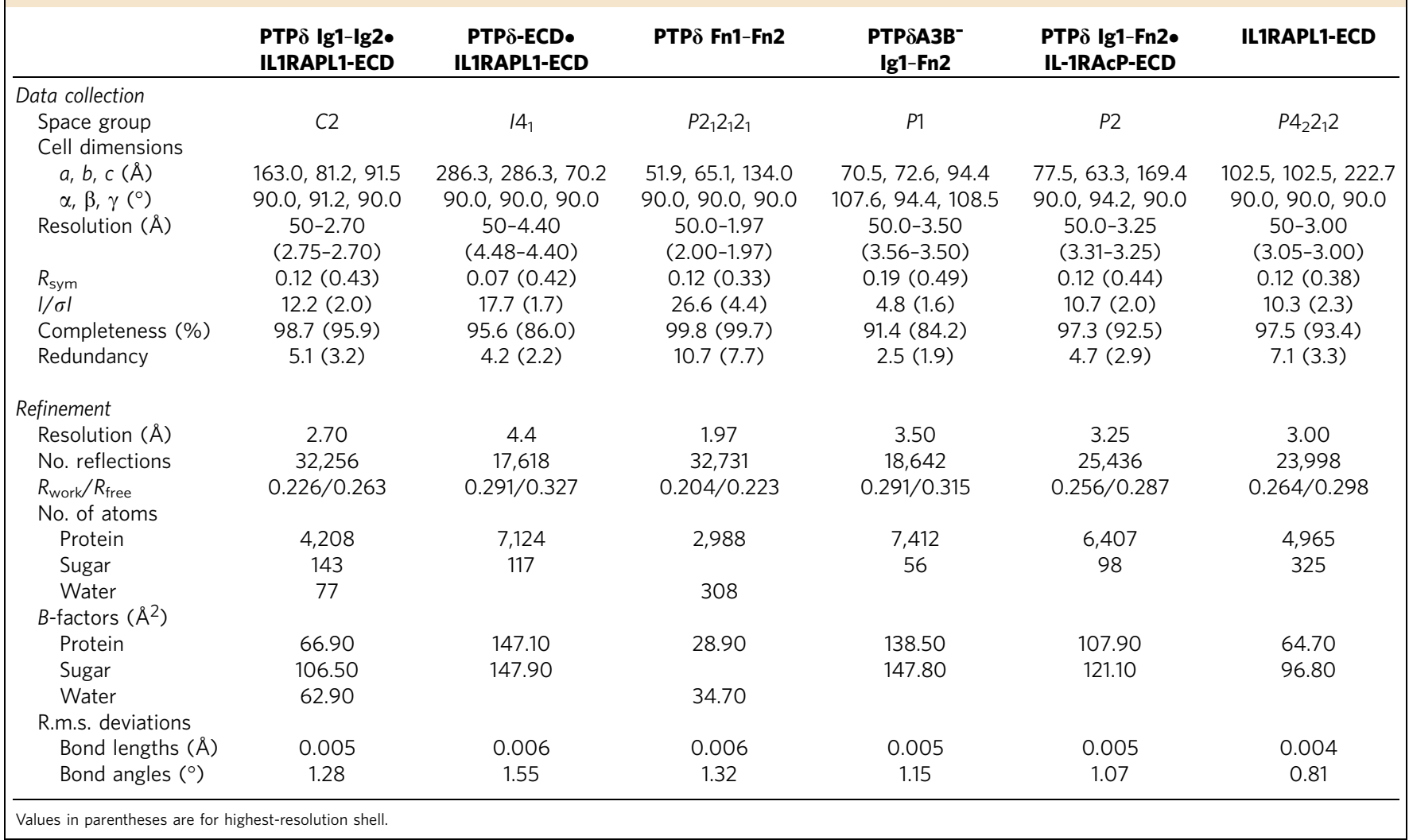

\section{Table 2 | Affinities $\left(K_{\mathrm{D}}\right)$ between the splicing variants of PTP $\delta$ and IL1RAPL1/IL-1RACP.}

\begin{tabular}{lcc} 
PTP $\boldsymbol{\delta}(\mathbf{m e A} / \mathbf{m e B})$ & IL1RAPL1 $(\boldsymbol{\mu} \mathbf{M})$ & IL-1RAcP $(\boldsymbol{\mu M} \mathbf{M})$ \\
\hline meA9/+ & $0.15 \pm 0.011$ & $0.68 \pm 0.013$ \\
meA9/- & $0.80 \pm 0.11$ & $2.0 \pm 0.085$ \\
meA6/+ & $0.81 \pm 0.14$ & $0.73 \pm 0.013$ \\
meA6/ - & ND & $2.3 \pm 0.016$ \\
meA3/+ & ND & $0.51 \pm 0.010$ \\
meA3/- & ND & $2.8 \pm 0.053$ \\
$-/+$ & ND & $2.2 \pm 0.075$ \\
$-/-$ & ND & $2.4 \pm 0.075$ \\
\hline ND, not detectable. & & \\
Data are presented as mean \pm s.d. & & \\
\hline
\end{tabular}

IL1RAPL1 decreased the affinity 11- and 16-fold, respectively (Table 3). The meA6-containing PTP $\delta$ variant also contains Arg196 but has a fivefold reduced affinity for IL1RAPL1 (Table 2). Therefore, this affinity reduction of the meA6-containing variant might be due to a lack of the concomitant hydrogen bonding. The atomic-level meA-specific interactions described here illustrate how the meA insertion contributes to the affinity between PTP $\delta$ and IL1RAPL1.

Relationship of meB with IL1RAPL1 Ig1-PTP $\delta$ Ig3 interaction. On the opposite side to the IL1RAPL1 Ig1-PTP $\delta$ Ig2 interface, the Ig1 domain of IL1RAPL1 interacts with the Ig3 domain of PTP $\delta$ with a buried surface area of $694 \AA^{2}$. The IL1RAPL1 Ig1PTP $\delta$ Ig 3 interface is stabilized by hydrophobic interactions (Fig. 1d). The side chains of IL1RAPL1 Tyr77 and PTP $\delta$ Tyr273 are aligned in an antiparallel manner to form a hydrophobic core, which is surrounded by Met75, Pro88 and Phe91 of IL1RAPL1
Table 3 | Affinities $\left(K_{D}\right)$ of PTP $\delta$ or IL1RAPL1 mutants for binding between PTP $\delta$ and IL1RAPL1.

\begin{tabular}{lc} 
& IL1RAPL1 $(\boldsymbol{\mu M})$ \\
\hline PTP $\delta$ & \\
WT & $0.15 \pm 0.011$ \\
R75A & $2.8 \pm 0.34$ \\
R196A & $1.7 \pm 0.45$ \\
Y273A & $0.86 \pm 0.11$ \\
meB & \\
GSSG & $0.14 \pm 0.022$ \\
QLEQ & $0.16 \pm 0.0026$ \\
ELREELRE & $0.15 \pm 0.052$ \\
ELREELREELRE & $0.42 \pm 0.038$ \\
& \\
& PTP $\boldsymbol{(}(\boldsymbol{\mu M})$ \\
\hline IL1RAPL1 & \\
WT & $0.15 \pm 0.011$ \\
W34A & ND \\
D37A & $2.4 \pm 0.016$ \\
M75A/Y77A/P88A/F91A & $2.8 \pm 0.48$ \\
D292A & $2.2 \pm 0.075$ \\
\hline ND, not detectable; WT, wild type. & \\
Data are presented as mean \pm s.d. & \\
\hline
\end{tabular}

and Pro270, Met271, Ile291 and Thr314 of PTP $\delta$. The Y273A mutation of PTP $\delta$ decreased the affinity for IL1RAPL1 sixfold, whereas the M75A/Y77A/P88A/F91A quadruple mutation of IL1RAPL1 decreased the affinity for PTP $\delta$ 19-fold (Table 3).

The meB insertion of PTP $\delta$ is important for binding between PTP $\delta$ and IL1RAPL1. The lack of meB reduces the affinity fivefold (Table 2). In the present PTP $\delta$-ECD•IL1RAPL1-ECD complex, the meB insertion appears to adjust the relative spacing 
and orientation between the Ig2 and Ig3 domains of PTP $\delta$ (Fig. 1b). When the apo structure of PTP $\delta$ Ig1-Fn2 containing meA3 and lacking meB $\left(\mathrm{PTP} \delta \mathrm{A}_{3} \mathrm{~B}^{-}\right.$) (Supplementary Fig. 1f), which we determined at $3.5 \AA$ resolution, is superposed onto the IL1RAPL1-ECD-bound structure of PTP $\delta$ Ig1-Fn2 (Supplementary Fig. 1g) using the Ig1-Ig2 unit of PTP $\delta$ as the reference, the Ig3 domain of the apo PTP $\delta \mathrm{AB}^{-}$is oriented in the opposite direction from that of the IL1RAPL1-ECD-bound PTP $\delta$ and cannot interact with IL1RAPL1, even if structural flexibility of the linker between the Ig2 and Ig3 domains of PTP $\delta$ is assumed (Supplementary Fig. 1g). Replacement of the native meB (Glu-Leu-Arg-Glu) by two tandem units of meB or a Gly-Ser-Ser-Gly or Gln-Leu-Glu-Gln tetrapeptide hardly affected the affinity, whereas that by three tandem units reduced the affinity threefold (Table 3). Insertions of four or more residues at the meB position are sufficient for binding to IL1RAPL1, but the longer insertion prevents the efficient binding. Therefore, the meB insertion likely acts as an adjustable linker to locate the Ig3 domain of PTP $\delta$ in the appropriate position for interacting with the Ig1 domain of IL1RAPL1, representing how the meB insertion contributes to the affinity between PTP $\delta$ and IL1RAPL1.

Interactions between IL1RAPL1 Ig3 and PTP $\delta$ Ig1 domains. The Ig3 domain of IL1RAPL1 interacts with the Ig1 domain of PTP $\delta$ with a buried surface area of $433 \AA^{2}$. The IL1RAPL1 Ig3-

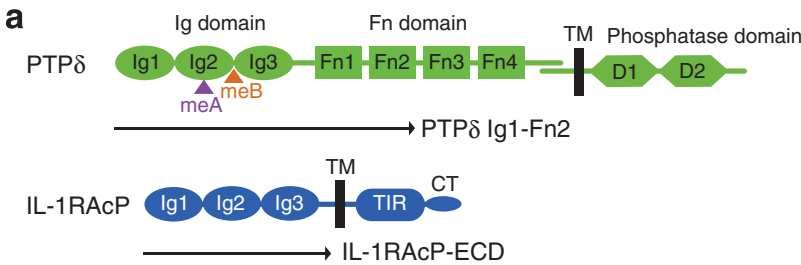

b

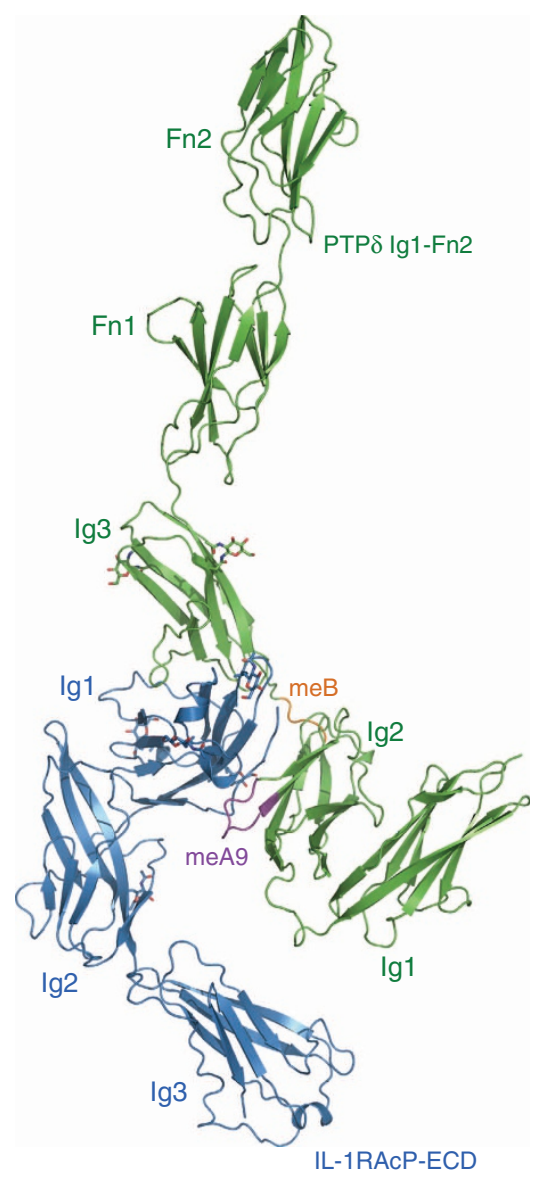

C

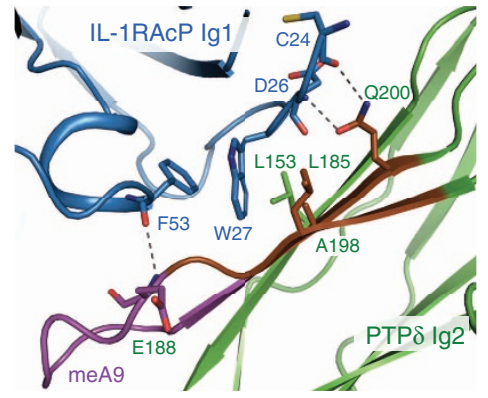

d

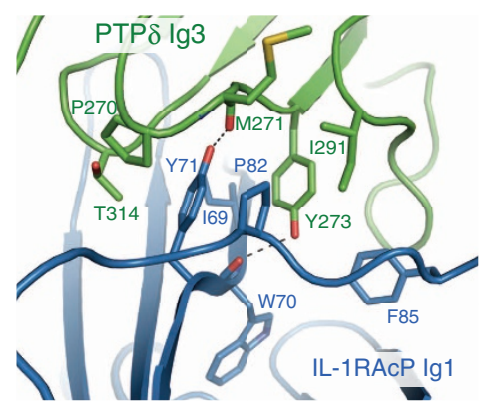

e

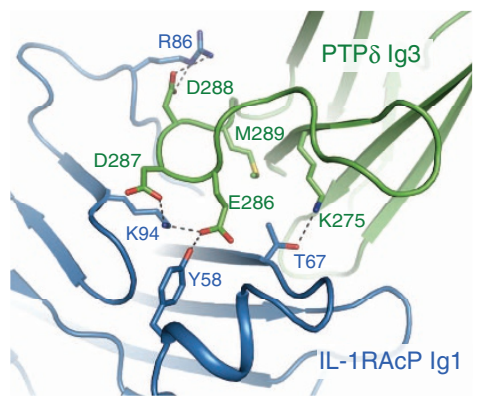

Figure 2 | Structure of the complex between PTP $\delta$ and IL-1RAcP. (a) Domain organizations of PTP $\delta$ and IL-1RAcP. The meA and meB insertion sites of PTP $\delta$ are indicated by magenta and orange triangles, respectively. (b) Overall structure of the PTP $\delta$ Ig1-Fn2•IL-1RAcP-ECD complex. PTP $\delta$ and IL-1RAcP are coloured green and blue, respectively. The meA9 and meB insertions are highlighted in magenta and orange, respectively. $N$-linked glycans are shown as sticks. (c) Interactions between the Ig1 domain of IL-1RAcP and the Ig2 domain of PTP $\delta$. The colouring scheme is the same as that in $\mathbf{b}$, except that IL1RAPL1/IL-1RAcP-Ig1-interacting $\beta$-strands of PTP $\delta$ are coloured brown. Residues involved in the interactions are shown as sticks. Hydrogen bonds are indicated by dotted lines. (d) Hydrophobic interactions between the Ig1 domain of IL-1RAcP and the Ig3 domain of PTP $\delta$. The representation scheme is the same as that in $\mathbf{c}$. (e) Hydrophilic interactions between the Ig1 domain of IL-1RAcP and the Ig3 domain of PTP $\delta$. The representation scheme is the same as that in $\mathbf{c}$ 
PTP $\delta$ Ig1 interface is overlapped with the putative binding pocket for glycosaminoglycan chains of heparan sulfate and chondroitin sulfate proteoglycans (Supplementary Fig. 1h), which mediate axonal growth control though the interaction with the type-IIa RPTPs $^{19}$. This third interface is formed primarily by hydrophilic interactions (Fig. 1e): a positively charged cluster comprising Arg75, Arg95 and Arg98 of PTP $\delta$ forms a hydrogen bond network with a negatively charged cluster comprising Glu291 and Asp292 of IL1RAPL1. This network is stabilized by a cation- $\pi$ interaction between Phe289 of IL1RAPL1 and Arg98 of PTP $\delta$. In addition, Arg124 of PTP $\delta$ forms a hydrogen bond with Glu337 of IL1RAPL1. To confirm the importance of this interface, IL1RAPL1 Asp292-PTPS Arg75 interacting pair was disrupted by Ala replacement. The D292A mutation of IL1RAPL1 and the R75A mutation of PTP $\delta$ reduced the affinity between IL1RAPL1 and PTP $\delta$ 15- and 19-fold, respectively (Table 3). When comparing the amino-acid sequences between IL1RAPL1 and IL-1RAcP, the Ig3 domain is the least conserved domain $(23 \%$ identity) among three Ig domains. As described below, the Ig3 domain of IL-1RAcP does not interact with PTP $\delta$ in the PTP $\delta \bullet I L-1 R A c P$ complex. Therefore, the negatively charged cluster of the IL1RAPL1 Ig3 domain and the positively charged cluster of the PTP $\delta$ Ig1 domain are another determinant for selective binding between PTP $\delta$ and IL1RAPL1.

Structure of the complex between PTP $\delta$ and IL-1RAcP. IL-1RAcP, a member of the same IL-1R family as IL1RAPL1 ( $\sim 30 \%$ sequence identity), also functions as a postsynaptic organizer for PTP $\delta$. However, the affinity of IL-1RAcP for PTP $\delta$ $\left(K_{\mathrm{d}}: 0.68 \mu \mathrm{M}\right)$ is fivefold lower than that of IL1RAPL1 (Table 2). The meA variation hardly affects the affinity. In addition, IL-1RAcP can bind to the PTP $\delta$ variant lacking both meA and meB $\left(K_{\mathrm{d}}: 2.4 \mu \mathrm{M}\right)$ in contrast to IL1RAPL1. To structurally explain these differences between IL1RAPL1 and IL-1RAcP, we also determined the crystal structure of IL-1RAcP-ECD in complex with PTP $\delta$ containing three Ig domains and two Fn domains (Ig1-Fn2) at $3.25 \AA$ resolution (Fig. 2a,b, Table 1). We had confirmed that PTP $\delta$ Ig1-Fn 2 efficiently binds to IL-1RAcP-ECD by testing various lengths of PTP $\delta$ (Supplementary Fig. 2a). The Ig1-Ig2 unit of PTP $\delta$ is insufficient for binding to IL-1RAcP. Similarly to the PTP $\delta \bullet I L 1 R A P L 1$ complex, the Ig1-Fn2 domains of PTP $\delta$ bind to IL-1RAcP-ECD at a ratio of 1:1 (Fig. 2b). The asymmetric unit contains one PTP $\delta$ IL-1RAcP complex (Supplementary Fig. 2b). The crystal packing patterns of the PTP $\delta \bullet I L 1 R A P L 1$ and PTP $\delta \bullet I L-1 R A c P$ complexes in this study are totally different from each other and not suggestive of their lateral associations in synapses. Although it can be assumed that activations of PTP $\delta$ and IL1RAPL1/IL-1RAcP are mediated by a ligand-induced monomer-dimer transition by analogy with those of receptor tyrosine kinases ${ }^{20-23}$ and Toll/IL-1-receptor families $^{24}$, our crystallographic and multiangle light scattering analyses using the ECDs of PTP $\delta$, IL-1RAcP and IL1RAPL1 could not support this activation mechanism (Supplementary Fig. 1b, 2b,c). The Ig1-Fn2 domains of PTP $\delta$ exhibit an elongated shape with a length of $160 \AA$ along the longest axis. Although overall shape of the IL-1RAcP-bound PTP $\delta$ Ig1-Fn2 resembles that of the IL1RAPL1-bound PTP $\delta$ Ig1-Fn2, the position and/or orientation of each domain are somewhat different between them. The Ig1-Ig3 domains of IL-1RAcP are arranged in an L-shape similar to those of IL1RAPL1, except that the relative orientation between the Ig1 and Ig2-Ig3 domains is changed by 20 degree (Supplementary Fig. 2d). Correspondingly, the Ig3 domain of IL-1RAcP does not interact with PTP $\delta$ in contrast to that of IL1RAPL1. Superposition between the Ig3 domains of IL1RAPL1 and IL-1RAcP shows that the binding surface of IL1RAPL1 for the Ig1 domain of PTP $\delta$ is not conserved in IL-1RAcP (Supplementary Fig. 2e).

Interactions between IL-1RAcP Ig1 and PTP $\delta$ Ig2. Similarly to the PTP $\delta$ IL1RAPL1 complex, interactions between the Ig1 domain of IL-1RAcP and the Ig2 domain of PTP $\delta$ occur on the IL1RAPL1/IL-1RAcP-Ig1-interacting $\beta$-strands (Fig. 2c). The interface between the IL-1RAcP Ig1 and PTP $\delta$ Ig2 domains comprises both hydrophobic and hydrophilic interactions with a buried surface area of $726 \AA^{2}$. Trp27 of IL-1RAcP hydrophobically interacts with Leu153, Ala198 and Leu185 of PTP $\delta$ (Fig. 2c). The W27A mutation of IL-1RAcP reduced the affinity sevenfold (Table 4). Although Arg196 of PTP $\delta$ is positioned in close proximity to Asp30 of IL-1RAcP, no observed electron density of its side chain guanidino group indicates that the interaction between Arg196 of PTP $\delta$ and Asp30 of IL-1RAcP is substantially weak (Supplementary Fig. 2f). Thus, the D30A mutation of IL-1RAcP and the R196A mutation of PTP $\delta$ hardly affected the affinity (Table 4). Basically, meA does not extensively interact with IL-1RAcP in the PTP $\delta$ Ig1-Fn2•IL-1RAcP-ECD complex, consistent with the finding that the variation of meA does not affect the affinity for IL-1RAcP (Table 2). Only one meA-mediated interaction is a hydrogen bond between the main-chain $\mathrm{N}$ atom of PTP $\delta$ Glu188 and the main-chain $\mathrm{O}$ atom of IL-1RAcP Phe53, which could be formed in the shorter mini-exon variants, meA6 and meA3, but not in the variant lacking meA. The complete deletion of meA shortens the IL1RAPL1/IL-1RAcP-Ig1-interacting $\beta$-strands, which might disturb and weaken the hydrophobic interaction of IL-1RAcP Trp27 with Leu153, Ala198 and Leu185 of PTP $\delta$, resulting in the threefold reduced affinity to IL-1RAcP (Table 2; compare meA9/ + with $-/+)$. These structural features may reflect that the meA insertion increases the affinity between PTP $\delta$ and IL-1RAcP, independently of its variation.

Relationship of meB with IL-1RAcP Ig1-PTP $\delta$ Ig3 interaction. The position and orientation of the PTP $\delta$ Ig 3 domain relative to the IL-1RAcP Ig1 domain are similar to those relative to the IL1RAPL1 Ig1 domain (Figs $1 \mathrm{~b}$ and $2 \mathrm{~b}$ ). The interface between

\begin{tabular}{|c|c|}
\hline & IL-1RAcP $(\mu M)$ \\
\hline \multicolumn{2}{|l|}{$\overline{P T P \delta}$} \\
\hline WT & $0.68 \pm 0.013$ \\
\hline R75A & $1.1 \pm 0.023$ \\
\hline R196A & $0.89 \pm 0.17$ \\
\hline Y273A & ND \\
\hline E286A & $5.3 \pm 0.52$ \\
\hline \multicolumn{2}{|l|}{ meB } \\
\hline GSSG & $0.64 \pm 0.036$ \\
\hline QLEQ & $0.91 \pm 0.019$ \\
\hline ELREELRE & $1.5 \pm 0.038$ \\
\hline \multirow[t]{2}{*}{ ELREELREELRE } & $3.8 \pm 0.21$ \\
\hline & PTP $\delta(\mu \mathrm{M})$ \\
\hline \multicolumn{2}{|l|}{ IL-1RACP } \\
\hline WT & $0.68 \pm 0.013$ \\
\hline W27A & $4.6 \pm 0.076$ \\
\hline D30A & $0.62 \pm 0.033$ \\
\hline K94A & $8.2 \pm 0.054$ \\
\hline 169A/Y71A/P82A/F85A & ND \\
\hline
\end{tabular}


the IL-1RAcP Ig1 and PTP $\delta$ Ig3 domains buries a surface area of $906 \AA^{2}$. The inside of the interface is stabilized by more tightly packed hydrophobic interactions than that of the IL1RAPL1 Ig1PTP $\delta$ Ig3 interface (Figs 1d and 2d). The side chains of IL-1RAcP Tyr71 and PTP $\delta$ Tyr273 are stacked in an antiparallel manner to form a hydrophobic core, which is surrounded by the side chains of IL-1RAcP Phe85, Pro82 and Ile69 and PTP 8 Pro270 and Ile291 and the aliphatic portion of PTP $\delta \mathrm{Thr} 314$. The side chains of IL-1RAcP Tyr71 and PTP $\delta$ Tyr273 are stabilized by hydrogen bonds with the main-chain $\mathrm{O}$ atoms of PTP $\delta$ Met271 and IL-1RAcP Trp70, respectively. Furthermore, the Ig1 domain of IL-1RAcP recognizes a negatively charged loop comprising Glu286, Asp287 and Asp288 of PTP $\delta$. Tyr58 and Arg86 of IL-1RAcP hydrogen bond with Glu286 and Asp288 of PTP $\delta$, respectively, whereas Lys94 of IL-1RAcP hydrogen bonds with both Glu286 and Asp287 of PTP $\delta$ (Fig. 2e). Conformation of this negatively charged loop is supported by Met289 of PTP $\delta$. In addition, Thr67 of IL-1RAcP hydrogen bonds with Lys275 of PTP $\delta$. Mutations of the IL-1RAcP Ig1-PTP $\delta$ Ig3 interface impaired the binding affinity more drastically than those of the IL1RAPL1 Ig1-PTP $\delta$ Ig3 interface: the Y273A mutation of PTP $\delta$ and the I69A/Y71A/P82A/F85A quadruple mutation of IL-1RAcP completely eliminated the binding ability, whereas the K94A mutation of IL-1RAcP and the E286A mutation of PTP $\delta$ decreased the affinity 12- and 8-fold, respectively (Table 4). Therefore, the interface mediated by PTP $\delta$ Ig 3 is more critically important for binding to IL-1RAcP than for binding to IL1RAPL1, consistent with the finding that PTP $\delta$ Ig3 is essential for binding to IL-1RAcP but not to IL1RAPL1 (Supplementary Figs $1 \mathrm{a}$ and $2 \mathrm{a}$ ).

Replacement of the native meB (Glu-Leu-Arg-Glu) by a Gly-Ser-Ser-Gly or Gln-Leu-Glu-Gln tetrapeptide hardly affected the affinity for IL-1RAcP, whereas that by two or three tandem units reduced the affinity two- or sixfold, respectively (Table 4). These impacts on the affinity for IL-1RAcP are similar to those for IL1RAPL1, supporting the notion that the meB insertion acts as an adjustable linker to control relative positions and orientations of the PTP $\delta$ Ig2 and Ig3 domains for their simultaneous interactions with the Ig1 domain of IL-1RAcP.

Synaptogenic activity. Finally, we investigated relationships between the structures and synaptogenic activity (that is, IL1RAPL1/IL-1RAcP-induced presynaptic differentiation and PTP $\delta$-induced postsynaptic differentiation). IL1RAPL1/IL1RAcP mutations were examined by fibroblast-neuron mixed culture assays (Fig. 3): HEK293T cells expressing IL1RAPL1/IL1RAcP mutants were co-cultured with cortical neurons and then, accumulation of a presynaptic protein Bassoon in the cortical neurons was analysed (Fig. 3a-d). Because IL1RAPL1- and IL-1RAcP-induced presynaptic differentiation is completely abolished and decreased by $\sim 70 \%$ in cortical neurons prepared from PTP $\delta$-deficient mice, respectively ${ }^{7,8}$, IL1RAPL1/IL-1RAcPinduced presynaptic differentiation is mostly mediated by the mixed, cognate PTP $\delta$ variants in neurons. On the other hand, to examine postsynapse-inducing activity of PTP $\delta$ mutants, magnetic beads conjugated with the ECDs of PTP $\delta$ mutants were co-cultured with cortical neurons, and accumulation of a postsynaptic protein Shank2 around the beads was analysed (Fig. 3e,f). PTP $\delta$-stimulating postsynaptic differentiation is mediated by IL1RAPL1, IL-1RAcP and possibly other unidentified postsynaptic organizer(s) in neurons.

Synaptogenic activities of IL1RAPL1 and IL-1RAcP mutants are basically correlated to their affinities to PTP $\delta$, which govern stimulation of the receptor activation and signal transmission inside cells. Synaptogenic activities of PTP $\delta$ mutants are also correlated with their affinities to IL1RAPL1. Remarkably, the
R75A mutation in the Ig1 domain of PTP $\delta$ reduced the synaptogenic activity to $\sim 7 \%$. This indicates that the interaction between the IL1RAPL1 Ig3 and PTP $\delta$ Ig1 domains is critically important for inducing postsynaptic differentiation. In contrast to the affinity of IL1RAPL1, that of IL-1RAcP is not so consistently correlated with synaptogenic activities of PTP $\delta$ mutants, possibly due to a lack of the interaction between the IL-1RAcP Ig3 and PTP $\delta$ Ig1 domains. Considering that IL-1RAcP knockout neurons retain nearly full activity of PTP $\delta$-induced postsynaptic differentiation, we speculate that the PTP $\delta \bullet I L-1 R A c P$ complex might function as a unidirectional organizer that is primarily responsible for inducing presynaptic differentiation in vivo, although IL-1RAcP can promote postsynaptic differentiation of cultured cortical neurons by binding to the meA3-containing PTP $\delta$ variant, which is specific to IL-1RAcP ${ }^{7}$. The Ig1 domain of PTP $\delta$ might be universally important for postsynaptic differentiation in vivo, which involves IL1RAPL1 and possibly other unidentified postsynaptic organizer(s).

\section{Discussion}

Recently, crystal structures of two different trans-synaptic type-IIa RPTP complexes have been reported ${ }^{14,25}$. One is the complex between Ig1-Ig2 of PTP $\sigma$ and leucine-rich repeat (LRR)-Ig1 of TrkC ${ }^{25}$, while the other is that between Ig1-Ig3 of PTP $\delta$ and the first LRR (LRR1) of Slitrk1 (ref. 14, Supplementary Fig. 3a). These and our type-IIa RPTP complex structures illustrate that the type-IIa RPTPs can be recognized by structurally distinct architectures. The TrkC- or Slitrk1interacting surface of the RPTPs is separated from the IL1RAPL1- or IL-1RAcP-interacting surface, except that a part of the TrkC-interacting surface is overlapped with the IL1RAPL1interacting surface. The conserved arginine residues in Ig1 of the RPTPs (that is, Arg95 and Arg98 of PTP $\delta$ and Arg96 and Arg99 of PTP $\sigma$ ) are recognized by both IL1RAPL1 and TrkC through similar hydrogen bond interactions (Supplementary Fig. 3b). Despite the separation of these binding surfaces of the RPTPs, pair-wise superposition between any two of these complex structures shows steric clashes between the bound postsynaptic partners, ruling out their simultaneous binding to the type-IIa RPTPs (Supplementary Fig. 3c). The IL1RAPL1- or IL-1RAcPinteracting residues of PTP $\delta$ are mostly conserved in the type-IIa RPTPs. Their differences in the specificity to IL1RAPL1 or IL-1RAcP appear to be dependent exclusively on the variation of meA and meB. Our previous analysis of Ptprs and Ptprf cDNA from the developing mouse brain suggested that none of the PTP $\sigma$ variants contains the mini-exon peptide insertion at the meA site ${ }^{8}$ and that $90 \%$ of LAR lacks the peptide insertion at the meB site $^{8}$. Therefore, IL1RAPL1 and IL-1RAcP induce synaptogenesis specifically through $\mathrm{PTP} \delta$, at least in the developing brain. On the other hand, the meB insertion is sufficient for binding to Slitrks. They may regulate synaptogenesis mainly through PTP $\sigma$ or PTP $\delta$ in the developing brain ${ }^{13,14}$, although they can potentially bind to all the type-IIa RPTP variants containing meB. Both the regulation of $\mathrm{meA}$ and $\mathrm{meB}$ choice by alternative splicing of the type-IIa RPTPs and the mutually exclusive binding among postsynaptic ligands for the type-IIa RPTPs may contribute to sharpening target specificity of central synaptogenesis.

The Ig-Ig interaction between cell-surface receptors is generally important for cell adhesion and communication in immune and neuronal systems. To our knowledge, binding between Ig-containing adhesion receptors is typically mediated by single, homotypic Ig-Ig interactions ${ }^{26-28}$ in neuronal systems. In contrast, binding between PTP $\delta$ and IL1RAPL1/IL-1RAcP is mediated by multiple, heterotypic Ig-Ig interactions. The meB insertion of PTP $\delta$ substantially contributes to these multiple, 
a

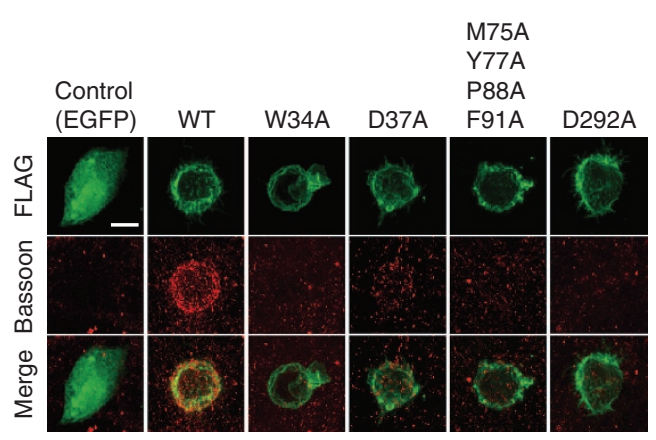

C

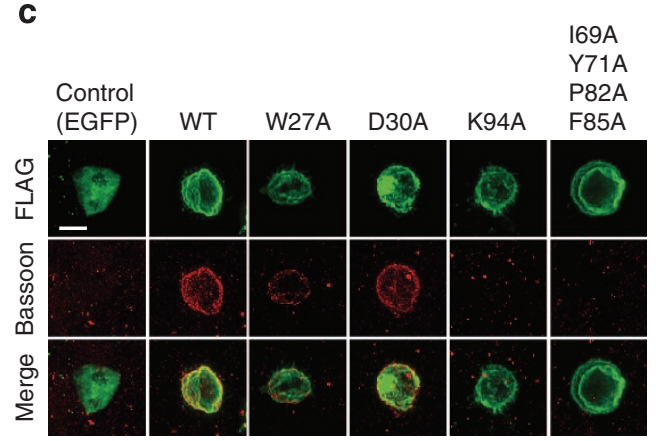

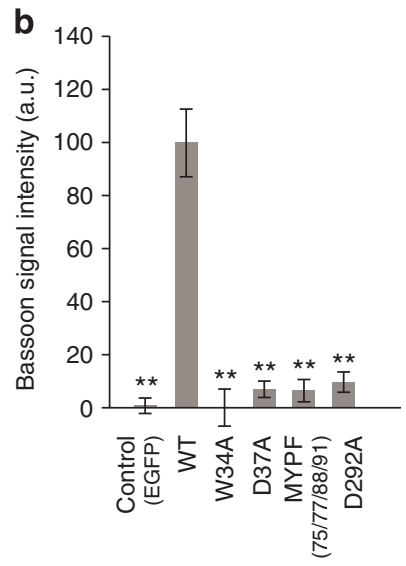

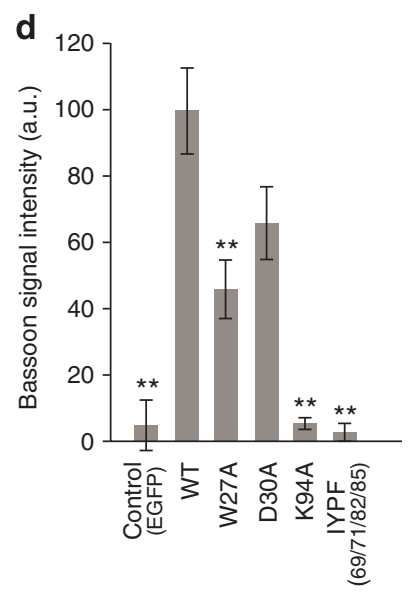

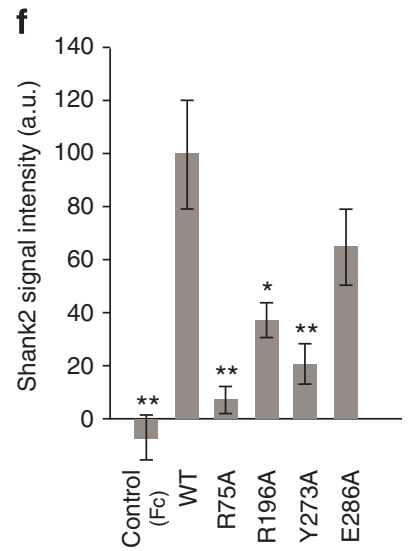

Figure 3 | Synaptogenic activities of IL1RAPL1, IL-1RAcP and PTP $\boldsymbol{\delta}$ mutants. (a,c) Accumulation of Bassoon signals (red) of cortical neurons co-cultured with HEK293T cells expressing FLAG-tagged IL1RAPL1 mutants (green) (a) and IL-1RAcP mutants (green) (c). (b,d) Intensity of staining signals for Bassoon on the surface of HEK293T cells expressing FLAG-tagged IL1RAPL1 mutants (b) and FLAG-tagged IL-1RAcP mutants (d) co-cultured with cortical neurons ( $n=16-19)$. (e) Accumulation of Shank2 signals (red) of cortical neurons co-cultured with magnetic beads conjugated with ECDs of PTP $\delta$ mutants. (f) Intensity of staining signals for Shank2 on the surface of magnetic beads conjugated with ECDs of PTP $\delta$ mutants co-cultured with cortical neurons $(n=27-38)$. All values represent mean \pm s.e.m. ${ }^{\star} P<0.05$ and ${ }^{* \star} P<0.01$ compared with HEK293T cells expressing wild-type IL1RAPL1 (b), wild-type IL-TRAcP (d) or wild-type PTP $\delta$-ECD-Fc coated beads (f); Tukey's test. Scale bars, $10 \mu \mathrm{m}(\mathbf{a}, \mathbf{c})$ and $5 \mu \mathrm{m}(\mathbf{e})$.

heterotypic Ig-Ig interactions; $\mathrm{meB}$ is located at the junction between the $\operatorname{Ig} 2$ and $\operatorname{Ig} 3$ domain of PTP $\delta$ and adjusts their relative spacing and orientation so that they can simultaneously interact with the Ig1 domain of IL-1RAcP or IL1RAPL1 (Fig. 4a). In the IL-1RAcP/IL1RAPL1-bound state, the Ig2 and Ig3 domains of PTP $\delta$ exhibits a unique conformation that differs from a linear or $\mathrm{V}$-shaped conformation, which is typically observed in a tandem repeat of Ig domains. Furthermore, this unique conformation of PTP $\delta$ Ig2-Ig3 also differs from the conformation in a Slitrk-bound state $^{14}$. Most PTP $\delta$ variants expressed in the developing mouse brain at postnatal day 11 contain meB and only $4 \%$ of variants lack $\mathrm{meB}^{8}$, suggesting that the meB-containing PTP $\delta$ variants should be mostly utilized for synaptogenesis in the brain. Therefore, the insertion of meB might contribute to allowing PTP $\delta$ Ig2-Ig3 to form some distinct conformations, depending on the structurally different postsynaptic ligands.

For binding of PTP $\delta$ to IL1RAPL1, either meA9 or meA6 is essential. Thus, the interface including meA is likely the primary interaction site. Accordingly, the W34A mutation of IL1RAPL1 (at the interface with $\mathrm{PTP} \delta$ Ig2) eliminated binding to $\mathrm{PTP} \delta$. 
a

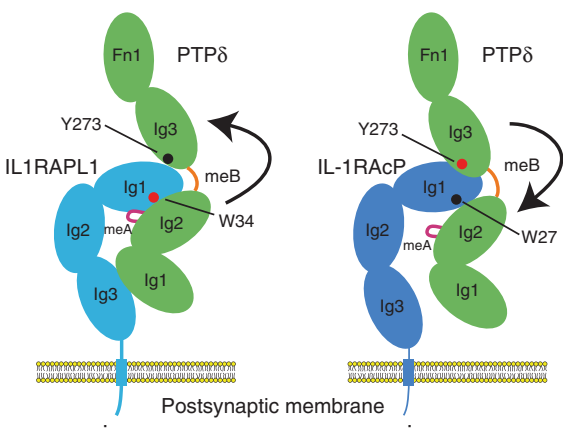

b

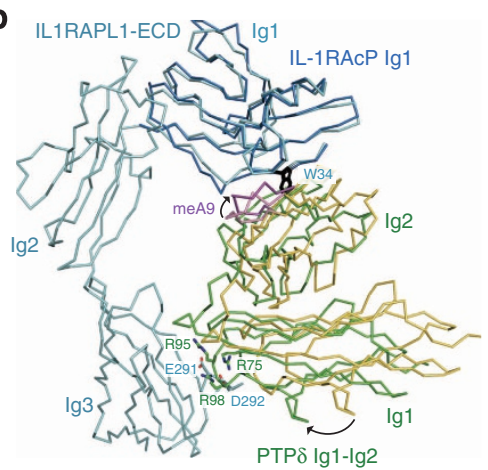

Figure 4 | Mechanistic insights into trans-synaptic adhesion between PTP $\boldsymbol{\delta}$ and IL1RAPL1 or IL-1RAcP. (a) Schematic drawing of the mechanism for the meA- and meB-regulated binding of PTP $\delta$ to IL1RAPL1 or IL-1RAcP. The colouring scheme is the same as those in Figs 1a and 2a. Red dots indicate the primary interaction site, whereas black dots indicate the secondary interaction site. Arrows indicate a model that the interaction at the primary site may be followed by the interaction at the secondary site. (b) Superposition of the lg1-lg2 unit in the IL-1RAcP-bound PTPS (yellow) on that in the IL1RAPL1bound PTP $\delta$ (green), using the Ig1 domain of IL1RAPL1/IL-1RAcP as the reference. Residues involved in the IL1RAPL1 Ig3-PTP $\delta$ Ig1 interface and the corresponding IL-1RAcP residues are shown as sticks.

Moreover, the interaction with meA appears coupled to the third interface involving PTP $\delta$ Ig1 and IL1RAPL1 Ig3, which is critical for binding between IL1RAPL1 and PTP $\delta$ and inducing bidirectional synaptic differentiation of cultured cortical neurons, as mentioned above. Comparison between the IL1RAPL1 and IL-1RAcP complexes exhibits a rotational difference in the orientations of the PTP $\delta$ Ig1-Ig2 unit, relative to the Ig1 domains of IL1RAPL1 and IL-1RAcP (Fig. 4b). Trp34 of IL1RAPL1 is positioned at the pivot for this rotation, which allows PTP $\delta$ Ig1 to interact with IL1RAPL1 Ig3. In this context, meA also contributes to the multiple, heterotypic Ig-Ig interactions between IL1RAPL1 and PTP $\delta$, besides meB, which assists in locating the Ig3 domain of PTP $\delta$ in the appropriate position for interacting with the Ig1 domain of IL1RAPL1. Consistent with this, the impact of the lack of meB on binding between PTP $\delta$ and IL1RAPL1 is comparable to that of the PTP $\delta$ Y273A mutation (deficient in the PTP $\delta$ Ig3-IL1RAPL1 Ig1 interaction). In contrast, the impact of the lack of meB on binding between PTP $\delta$ and IL-1RAcP is incompatible to that of the PTP $\delta$ Y273A mutation, but rather is comparable to that of mutations at the IL-1RAcP Ig1-PTP $\delta$ Ig2 interface. The W27A mutation of IL-1RAcP, which is equivalent to the W34A mutation of IL1RAPL1, reduced the affinity sevenfold but did not eliminate the binding. Therefore, for binding to IL-1RAcP, the PTP $\delta$ Ig3-IL-1RAcP Ig1 interface is likely the primary interaction site, and the meB insertion may serve as the adjustable linker to locate the Ig2 domain of PTP $\delta$ in the appropriate position for interacting with the Ig1 domain of IL-1RAcP (Fig. 4a).

IL-1RAcP functions in both immune and neuronal systems, whereas IL1RAPL1 is exclusively expressed in neuronal systems, suggesting that IL1RAPL1 has evolved to be more specialized for neuronal systems than IL-1RAcP. Correspondingly, IL-1RAcP binds to PTP $\delta$ weaker than IL1RAPL1 but has a broader specificity to the meA variants. In contrast, IL1RAPL1 has more strict specificity to meA and can bind to PTP $\delta$ more tightly than IL-1RAcP. The affinity difference generated by the splice-insert signaling codes is substantially high for binding between PTP $\delta$ and IL1RAPL1 but modest for binding between PTP $\delta$ and IL-1RAcP. On the other hand, in our synaptogenic assays, the strength of the induced synaptic differentiation seems non-linearly correlated with the affinity; rather, there seems to be the threshold of the affinity for inducing the synaptic differentiation, which might contribute to controlling appropriate synaptic target selection to avoid misconnections to target cells with low affinity ligands. Further functional studies including identification and characterization of additional regulatory factors are awaited to discuss the activation and signaling mechanisms of IL1RAPL1/IL-1RAcP and PTP $\delta$ for inducing synaptic differentiation.

\section{Methods}

Protein expression and purification. Genes encoding mouse PTP $\delta$-ECD, PTP $\delta$ Ig1-Fn4, PTP $\delta$ Ig1-Fn3, PTP $\delta$ Ig1-Fn2, PTP $\delta$ Ig1-Fn1, PTP $\delta$ Ig1-Ig3 and PTP $\delta$ Ig1-Ig2 (residues 28-861, 28-699, 28-611, 28-518, 28-418, 28-325 and 28-237, respectively) were amplified from cDNA (accession No. NM_011211.3) by PCR and cloned into pEBMulti-Neo vector (Wako Pure Chemical Industries) with $\mathrm{N}$-terminal signal sequences derived from pHLsec vector and C-terminal hexahistidine tag ${ }^{29}$. PTP $\delta \mathrm{A}_{3} \mathrm{~B}^{-}$Ig1-Fn2 gene was also cloned from $\mathrm{CDNA}^{8}$ in the same manner as the PTP $\delta$ constructs. Genes encoding mouse IL-1RAcP-ECD (residues 21-351, gene accession No. NM_008364.2) and IL1RAPL1-ECD (residues 19-352, gene accession No. NM_001160403.1) were cloned into pEBMulti-Neo vector, with $\mathrm{N}$-terminal Igא signal sequence and C-terminal hexahistidine tag ${ }^{7,8}$. All proteins were transiently expressed using Freestyle 293-F cells (Invitrogen). For crystallography, proteins were purified from 0.2 to 11 of culture media by Ni-NTA (Qiagen) or Talon metal affinity resin (Clontech) with a standard protocol and dialysed against $20 \mathrm{mM}$ Tris- $\mathrm{HCl}$ buffer ( $\mathrm{pH}$ 7.5) containing $150 \mathrm{mM} \mathrm{NaCl}$. Proteins were concentrated at 5-10 gl ${ }^{-1}$, flash-frozen in liquid $\mathrm{N}_{2}$ and stored at $-80^{\circ} \mathrm{C}$ until use. A gene encoding PTP $\delta$ (Fn1-Fn2) (residues 328-518) was cloned into pET21a vector (Novagen). PTP $($ Fn1-Fn2) was overexpressed in Rosetta (DE3) Escherichia coli cells (Novagen) and purified by the $\mathrm{Ni}$ affinity chromatography followed by the size exclusion chromatography with Superdex 200 16/60 (GE Healthcare).

Pull-down assay. Binding abilities of serially deleted PTP $\delta$-ECDs were tested by pull-down assay with the C-terminally Fc-tagged IL-1RAcP- or IL1RAPL1-ECD (IL-1RAcP- or IL1RAPL1-Fc, respectively). IL-1RAcP- and IL1RAPL1-Fc were purified by Protein G Sepharose (GE Healthcare) ${ }^{7,8}$. The purified IL-1RAcP- or IL1RAPL1-Fc was mixed with the PTP $\delta$ proteins at 1:1 molar ratio and then immobilized with Protein G Sepharose beads (GE Healthcare). After washing by phosphate-buffered saline (PBS), the bound protein complexes were eluted by SDS sample loading buffer, followed by SDS-PAGE analyses.

Crystallization. PTP $\delta A 3 B^{-}$Ig1-Fn2, PTP $\delta$ Fn1-Fn2, IL1RAPL1 and the PTP $\delta$ Ig1-Fn2•IL-1RAcP-ECD, PTP $\delta$-ECD•IL1RAPL1-ECD and PTP $\delta$ Ig1-Ig2• IL1RAPL1-ECD complexes were crystallized by the sitting drop vapour diffusion method. Protein solutions were mixed with the following reservoir solutions: $10 \%$ polyethylene glycol (PEG) 3350, $0.1 \mathrm{M}$ ammonium iodide for PTP $\delta \mathrm{A} 3 \mathrm{~B}^{-}$ Ig1-Fn2; 20\% PEG3350, $0.1 \mathrm{M} \mathrm{MgCl}_{2}$ and $0.1 \mathrm{M}$ BisTris (pH 5.5) for PTP $\delta$ Fn1-Fn2; 2.0-2.4 M ammonium sulfate for IL1RAPL1; 15\% PEG3350, $0.1 \mathrm{M}$ ammonium sulfate and $0.1 \mathrm{M}$ tri-sodium citrate ( $\mathrm{pH}$ 5.5) for the PTP $\delta$ Ig1-Fn2•IL-1RAcP-ECD complex; 12\% PEG4000, 0.1 M lithium sulfate, $0.1 \mathrm{M}$ ADA (pH 6.5) for the PTP $\delta$-ECD•IL1RAPL1-ECD complex; 15\% PEG4000, 0.1 M MES (pH 6.0) for the PTP $\delta$ Ig1-Ig2•IL1RAPL1-ECD complex. For the crystallization of the complexes, two protein samples were mixed at a molar ratio of 1:1 to a final concentration of $\sim 6 \mathrm{gl}^{-1}$ before crystallization experiments. All 
crystallization experiments except for PTP $\delta$ Fn1-Fn2 were performed at $20^{\circ} \mathrm{C}$. Temperature shift from 4 to $28^{\circ} \mathrm{C}$ drastically improved the crystal size of PTP $\delta$ Fn1-Fn2. Crystals were flash-frozen in liquid $\mathrm{N}_{2}$, followed by soaking in the reservoir solutions supplemented with the following cryo-protectants: $25 \%$ glycerol for PTP $\delta \mathrm{A}_{3} \mathrm{~B}^{-}$Ig1-Fn2 and the PTP $\delta$ Ig1-Ig2•IL1RAPL1-ECD complex; $25 \%$ ethylene glycol for PTP $\delta$ Fn1-Fn2; 27\% ethylene glycol for IL1RAPL1; 35\% xylitol for the PTP Ig1-Fn2・IL-1RAcP-ECD complex; 20\% ethylene glycol for the PTP $\delta$-ECD॰IL1RAPL1-ECD complex.

Crystallography. All data were collected at $100 \mathrm{~K}$ at BL41XU in SPring-8, and processed with HKL2000 (ref. 30) and CCP4 program suite ${ }^{31}$. Data collection and refinement statistics were summarized in Table 1 . Resolutions were estimated, basically based on $I / \sigma I$ values $(\sim 2)$. To improve the quality of the atomic models, the resolutions of PTP $\delta$-ECD $\bullet I L 1 R A P L 1-E C D$ and PTP $\delta A_{3 B}{ }^{-}$Ig1-Fn2 were set to be as high as possible. The resolution of PTP $\delta$ Fn1-Fn2 was limited by the size of the detector. We first determined a crystal structure of the PTP $\delta$ Ig1-Ig2•IL1RAPL1-ECD complex by the molecular replacement method using IL-1RAcP-ECD (PDB:4DEP, 3O4O) ${ }^{32,33}$ and PTP $\delta$ Ig1-Ig2 (PDB:2YD6) ${ }^{19}$ as the search models with the program Molrep ${ }^{34}$. Model building and refinement were carried out using the programs $\operatorname{Coot}^{35}$ and Phenix ${ }^{36}$, respectively. The final model of the PTP $\delta$ Ig1-Ig2•IL1RAPL1-ECD complex was refined at $2.7 \AA$ to $R_{\text {work }}$ and $R_{\text {free }}$ values of 22.6 and $26.3 \%$, respectively. Then, the PTP $\delta$-ECD $\bullet I L 1 R A P L 1-E C D$ complex structure was determined by the molecular replacement method using IL1RAPL1-ECD in the PTP $\delta$ Ig1-Ig2-bound state, PTP $\sigma$ Ig3 (PDB:2YD9) ${ }^{19}$, LAR Fn2 (PDB:2DJU) and PTP $\delta$ Fn2 (PDB:2DLH) as the search models. The final model of the PTP $\delta$-ECD•IL1RAPL1-ECD complex was refined at $4.4 \AA$ to $R_{\text {work }}$ and $R_{\text {free }}$ values of 29.1 and $32.7 \%$, respectively. In addition, $2.0-\AA$-resolution structure of PTP $\delta$ Fn1-Fn2 was solved by the molecular replacement method and refined with the program Phenix to $R_{\text {work }}$ and $R_{\text {free }}$ values of 20.4 and $22.3 \%$, respectively. The structure of IL1RAPL1-ECD was determined by the molecular replacement method and refined at $3.0 \AA$ to $R_{\text {work }}$ and $R_{\text {free }}$ values of 26.4 and $29.8 \%$, respectively. The structure of PTP $\delta$ Igl-Fn $2 \bullet I L-1$ RAcP-ECD complex was determined by the molecular replacement method using IL-1RAcP-ECD (PDB:4DEP, 3O4O) ${ }^{32,33}$, PTP $\delta$ Ig1-Fn2 (PDB:2YD6) $)^{19}$ and our refined PTP $\delta$ Fn1-Fn2 structure as the search models. The final model of the PTP $\delta$ Ig1-Fn2•IL1RAcP-ECD complex was refined at $3.25 \AA$ to $R_{\text {work }}$ and $R_{\text {free }}$ values of 25.6 and $28.7 \%$, respectively. The structure of $\mathrm{PTP} \delta \mathrm{A} 3 \mathrm{~B}^{-} \mathrm{Ig} 1-\mathrm{Fn} 2$ was determined by the molecular replacement method and refined at $3.5 \AA$ to $R_{\text {work }}$ and $R_{\text {free }}$ values of 29.1 and $31.5 \%$, respectively. The buried surface area was calculated with the program PISA $^{37}$. The stereochemistry of the final model was assessed by the program Procheck. Percentages of residues in the most favoured regions of the Ramachandran plot are $87.9 \%, 81.2 \%, 86.7 \%, 89.4 \%, 88.1 \%$ and $89.1 \%$ for PTP $\delta$ Ig1-Ig2•IL1RAPL1-ECD, PTP $\delta$-ECD•IL1RAPL1-ECD, PTP $\delta$ Fn1-Fn2, PTP $\delta A_{3} B^{-}$Ig1-Fn2, PTP $\delta$ Ig1-Fn2•IL-1RAcP-ECD and IL1RAPL1-ECD, respectively. No residues are in the disallowed regions for all these structures. Structural figures were prepared with the program PyMol (Schrödinger, LLC).

SPR analysis. SPR equilibrium experiments were carried out by using Biacore T200 (GE Healthcare) at $25^{\circ} \mathrm{C}$ in $10 \mathrm{mM}$ Hepes (pH 7.9) containing $150 \mathrm{mM}$ $\mathrm{NaCl}, 0.05 \%$ Tween-20. IL1RAPL1-ECD-His 6 was immobilized on a CM5 sensor chip by the amine-coupling method at a density of about 2,000 resonance units (RU), whereas IL-1RAcP-ECD-Fc was captured at a density of about $700 \mathrm{RU}$ by anti-Fc antibodies that were immobilized on a CM5 sensor chip. Splicing variants or mutants of PTP $\delta$ Ig1-Fn1 were injected as the analytes with the concentration range from 15.6 to $4,000 \mathrm{nM}$. The IL1RAPL1-immobilized sensor chip was regenerated by $10 \mathrm{mM} \mathrm{NaOH}$. The sensorgrams are shown in Supplementary Fig. 4.

Cell cultures and co-culture assay. A central nervous system-restricted isoform of IL-1RAcP, IL-1RAcPb, was used for HEK293T cell-based analyses of IL-1RAcP mutants. Primary cortical cultures were prepared from mice at E18 (ref. 38). The cortical cells were placed on coverslips coated with $30 \mu \mathrm{g} \mathrm{ml}^{-1}$ poly-L-lysine and $10 \mu \mathrm{g} \mathrm{ml}^{-1}$ mouse laminin at the density of $5 \times 10^{5}$ cells per well for co-culture assay. The cells were cultured in Neurobasal-A supplemented with $2 \%$ B-27 supplement (Invitrogen), $5 \% \mathrm{FCS}, 100 \mathrm{U} \mathrm{ml}^{-1}$ penicillin, $100 \mu \mathrm{g} \mathrm{ml}^{-1}$ streptomycin and $0.5 \mathrm{mM}$ L-glutamine for $24 \mathrm{~h}$, and then cultured in the same medium without FCS. Cultures of HEK293T cells were maintained in DMEM supplemented with $10 \% \mathrm{FCS}^{38}$. Expression vectors for mutated forms of PTP $\delta$ ECD-Fc, FLAG-tagged IL-1RAcPb and FLAG-tagged IL1RAPL1 were generated by PCR-based mutagenesis using pEB6-PTP $\delta$-ECD-Fc, pFLAG-IL-1RAcPb and pFLAG-IL1RAPL1 (ref. 7) as templates, respectively. Expression vectors for FLAGtagged IL1RAPL1 and IL-1RAcP mutants were transfected to HEK293T cells using NanoJuice transfection reagent (Novagen). After 2 days of culture, the transfected cells were washed with PBS containing $2 \mathrm{mM}$ EDTA, and incubated with the same buffer at $37^{\circ} \mathrm{C}$ for $10 \mathrm{~min}$. Dispersed cells were plated onto cortical neurons at days in vitro (DIV) 8 . After $24 \mathrm{~h}$ of co-culture, cells were fixed for immunostaining with mouse anti-Bassoon (Stressgen, 1:300) and rabbit anti-FLAG (Sigma, 1:1,000) antibodies. Fc and ECDs of PTP $\delta$ mutant proteins fused to Fc in HEK293T cell culture medium were bound to Protein A-conjugated magnetic particles (smooth surface, $4.0-4.5 \mu \mathrm{m}$ diameter; Spherotech). Beads coupled with Fc or Fc fusion proteins were added to cortical neurons (DIV 14). After $24 \mathrm{~h}$, cultures were fixed for immunostaining with rabbit anti-Shank2 antibody (Frontier Institute, 1:200).

Image acquisition and quantification. Images of fibroblast-neuron or beadneuron co-culture experiments were acquired with a confocal laser-scanning microscope TCS SP5II (Leica) under constant conditions as to laser power, iris, gain, $z$-steps and zoom setting throughout the experiments. The images were collected from at least two separate cell cultures. All quantitative measurements were performed with Image $1.36 \mathrm{~b}$ software ${ }^{39}$. The intensities of immunostaining signals for Bassoon or Shank2 were measured as the optical mean density within circles of 30- and 7- $\mu \mathrm{m}$ diameters enclosing transfected HEK293T cells and coated-beads, respectively. Statistical significance was evaluated by one-way analysis of variance followed by post hoc Tukey's test.

\section{References}

1. Carrie, A. et al. A new member of the IL-1 receptor family highly expressed in hippocampus and involved in X-linked mental retardation. Nat. Genet. 23, 25-31 (1999).

2. Jamain, S. et al. Mutations of the X-linked genes encoding neuroligins NLGN3 and NLGN4 are associated with autism. Nat. Genet. 34, 27-29 (2003).

3. Sudhof, T. C. Neuroligins and neurexins link synaptic function to cognitive disease. Nature 455, 903-911 (2008).

4. Pinto, D. et al. Functional impact of global rare copy number variation in autism spectrum disorders. Nature 466, 368-372 (2010).

5. Takahashi, H. \& Craig, A. M. Protein tyrosine phosphatases $\mathrm{PTP} \delta, \mathrm{PTP} \sigma$, and LAR: presynaptic hubs for synapse organization. Trends Neurosci. 36, 522-534 (2013).

6. Um, J. W. \& Ko, J. LAR-RPTPs: synaptic adhesion molecules that shape synapse development. Trends Cell Biol. 23, 465-475 (2013).

7. Yoshida, T. et al. Interleukin-1 receptor accessory protein organizes neuronal synaptogenesis as a cell adhesion molecule. J. Neurosci. 32, 2588-2600 (2012).

8. Yoshida, T. et al. IL-1 receptor accessory protein-like 1 associated with mental retardation and autism mediates synapse formation by trans-synaptic interaction with protein tyrosine phosphatase $\delta$. J. Neurosci. 31, 13485-13499 (2011).

9. Takahashi, H. et al. Postsynaptic TrkC and presynaptic PTP $\sigma$ function as a bidirectional excitatory synaptic organizing complex. Neuron 69, 287-303 (2011).

10. Woo, J. et al. Trans-synaptic adhesion between NGL-3 and LAR regulates the formation of excitatory synapses. Nat. Neurosci. 12, 428-437 (2009).

11. Kwon, S. K., Woo, J., Kim, S. Y., Kim, H. \& Kim, E. Trans-synaptic adhesions between netrin-G ligand-3 (NGL-3) and receptor tyrosine phosphatases LAR, protein-tyrosine phosphatase $\delta(\mathrm{PTP} \delta$ ), and $\mathrm{PTP} \sigma$ via specific domains regulate excitatory synapse formation. J. Biol. Chem. 285, 13966-13978 (2010).

12. Takahashi, H. et al. Selective control of inhibitory synapse development by Slitrk3-PTP $\delta$ trans-synaptic interaction. Nat. Neurosci. 15, 389-398 S1-S2 (2012).

13. Yim, Y. S. et al. Slitrks control excitatory and inhibitory synapse formation with LAR receptor protein tyrosine phosphatases. Proc. Natl Acad. Sci. USA 110, 4057-4062 (2013).

14. Um, J. W. et al. Structural basis for LAR-RPTP/Slitrk complex-mediated synaptic adhesion. Nat. Commun. 5, 5423 (2014).

15. Uemura, T. et al. Trans-synaptic interaction of GluR $\delta 2$ and Neurexin through Cbln1 mediates synapse formation in the cerebellum. Cell 141, 1068-1079 (2010).

16. Siddiqui, T. J., Pancaroglu, R., Kang, Y., Rooyakkers, A. \& Craig, A. M. LRRTMs and neuroligins bind neurexins with a differential code to cooperate in glutamate synapse development. J. Neurosci. 30, 7495-7506 (2010).

17. Koehnke, J. et al. Splice form dependence of $\beta$-neurexin/neuroligin binding interactions. Neuron 67, 61-74 (2010).

18. Wei, Z. \& Zhang, M. A structural approach to decipher the neurexin and neuroligin splice isoform code. Neuron 67, 1-2 (2010).

19. Coles, C. H. et al. Proteoglycan-specific molecular switch for RPTP $\sigma$ clustering and neuronal extension. Science 332, 484-488 (2011).

20. Tonks, N. K. Protein tyrosine phosphatases: from genes, to function, to disease. Nat. Rev. Mol. Cell Biol. 7, 833-846 (2006).

21. Groves, J. T. \& Kuriyan, J. Molecular mechanisms in signal transduction at the membrane. Nat. Struct. Mol. Biol. 17, 659-665 (2010).

22. Lemmon, M. A. \& Schlessinger, J. Cell signaling by receptor tyrosine kinases. Cell 141, 1117-1134 (2010).

23. Dabrowski, A. \& Umemori, H. Orchestrating the synaptic network by tyrosine phosphorylation signalling. J. Biochem. 149, 641-653 (2011).

24. Ferrao, R., Li, J., Bergamin, E. \& Wu, H. Structural insights into the assembly of large oligomeric signalosomes in the Toll-like receptor-interleukin-1 receptor superfamily. Sci. Signal. 5, re3 (2012). 
25. Coles, C. H. et al. Structural basis for extracellular cis and trans RPTP $\sigma$ signal competition in synaptogenesis. Nat. Commun. 5, 5209 (2014).

26. Aricescu, A. R. \& Jones, E. Y. Immunoglobulin superfamily cell adhesion molecules: zippers and signals. Curr. Opin. Cell Biol. 19, 543-550 (2007).

27. Wei, C. H. \& Ryu, S. E. Homophilic interaction of the L1 family of cell adhesion molecules. Exp. Mol. Med. 44, 413-423 (2012)

28. Samanta, D. \& Almo, S. C. Nectin family of cell-adhesion molecules: structural and molecular aspects of function and specificity. Cell Mol. Life Sci. 72, 645-658 (2015).

29. Aricescu, A. R., Lu, W. \& Jones, E. Y. A time- and cost-efficient system for high-level protein production in mammalian cells. Acta Crystallogr. D 62, 1243-1250 (2006).

30. Otwinowski, Z. \& Minor, W. Processing of X-ray diffraction data collected in oscillation mode. Methods Enzymol. 276, 20 (1997).

31. Winn, M. D. et al. Overview of the CCP4 suite and current developments. Acta Crystallogr. D Biol. Crystallogr. 67, 8 (2011).

32. Thomas, C., Bazan, J. F. \& Garcia, K. C. Structure of the activating IL-1 receptor signaling complex. Nat. Struct. Mol. Biol. 19, 455-457 (2012).

33. Wang, D. et al. Structural insights into the assembly and activation of IL-1 $\beta$ with its receptors. Nat. Immunol. 11, 905-911 (2010).

34. Vagin, A. T. A. MOLREP: an automated program for molecular replacement. J. Appl. Cryst. 30, 4 (1997).

35. Emsley, P. \& Cowtan, K. Coot: model-building tools for molecular graphics. Acta Crystallogr. D 60, 2126-2132 (2004).

36. Adams, P. D. et al. The Phenix software for automated determination of macromolecular structures. Methods 55, 94-106 (2011).

37. Krissinel, E. \& Henrick, K. Inference of macromolecular assemblies from crystalline state. J. Mol. Biol. 372, 774-797 (2007).

38. Uemura, T., Mori, H. \& Mishina, M. Direct interaction of GluR $\delta 2$ with Shank scaffold proteins in cerebellar Purkinje cells. Mol. Cell Neurosci. 26, 330-341 (2004).

39. Uemura, T. \& Mishina, M. The amino-terminal domain of glutamate receptor $\delta 2$ triggers presynaptic differentiation. Biochem. Biophys. Res. Commun. 377, 1315-1319 (2008)

\section{Acknowledgements}

We thank the beam-line staff at NW12A, BL-1A, BL-5A and BL17A of Photon Factory (Tsukuba, Japan) and BL32XU and BL41XU of SPring-8 (Hyogo, Japan) for technical help during data collection. This work was supported by Grant-in-Aid for Scientific Research on Innovative Areas 22121003 (S.F.), 22123008 (M.M.), 25110708 (T.Y.), 25121710 (T.U.), Grant-in-Aid for Scientific Research (A) 21249012, 24249014 (M.M.) Grant-in-Aid for Scientific Research (B) 25293057 (T.Y), 25290021 (T.U.), Grant-in-Aid for Scientific Research (C) 24570126 (A.Y.), Grant-in-Aid for challenging Exploratory Research 26640038 (T.Y.), 26640039 (T.U.), PRESTO, JST (T.Y.) and CREST, JST (S.F. and T.U.).

\section{Author contributions}

T.Y., A.M., T.S. and A.Y. performed pull-down assays and crystallization. A.Y., S.F., Y.S and S.G.-I. collected diffraction data. A.Y. and S.F. analysed the collected data and determined the structures. T.Y., T.S. and S.I.-O. performed cell biological experiments. A.Y., T.Y. and S.F. wrote the paper with editing by T.U., H.M. and M.M. T.Y., M.M. and S.F. designed and supervised the study.

\section{Additional information}

Accession codes: Atomic coordinates and structure factors have been deposited with the accession codes 4YFC (PTP $\delta$ Ig1-Ig2•IL1RAPL1-ECD), 4YFD (PTP $\delta$ Ig1-Fn2•IL-

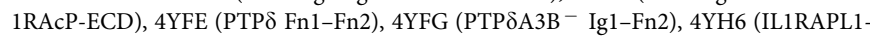
$\mathrm{ECD})$ and $4 \mathrm{YH} 7(\mathrm{PTP} \delta$-ECD・IL1RAPL1-ECD).

Supplementary Information accompanies this paper at http://www.nature.com/ naturecommunications

Competing financial interests: The authors declare no competing financial interests.

Reprints and permission information is available online at http://npg.nature.com/ reprintsandpermissions/

How to cite this article: Yamagata, A. et al. Mechanisms of splicing-dependent transsynaptic adhesion by PTP $\delta$-IL1RAPL1/IL-1RAcP for synaptic differentiation. Nat. Commun. 6:6926 doi: 10.1038/ncomms7926 (2015).

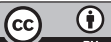

This work is licensed under a Creative Commons Attribution 4.0 International License. The images or other third party material in this article are included in the article's Creative Commons license, unless indicated otherwise in the credit line; if the material is not included under the Creative Commons license, users will need to obtain permission from the license holder to reproduce the material To view a copy of this license, visit http://creativecommons.org/licenses/by/4.0/ 